University of Wollongong

Research Online

Faculty of Engineering and Information

Faculty of Engineering and Information

Sciences - Papers: Part B

Sciences

2018

\title{
Effect of multi-walled carbon nanotubes on the cross-linking density of the poly(glycerol sebacate) elastomeric nanocomposites
}

\author{
Yi Yan \\ University of Wollongong, yy619@uowmail.edu.au \\ Vitor Sencadas \\ University of Wollongong, victors@uow.edu.au \\ Tiantian Jin \\ University of Wollongong, tj122@uowmail.edu.au \\ Xu-Feng Huang \\ University of Wollongong, xhuang@uow.edu.au \\ Wilford Lie \\ University of Wollongong, wilford@uow.edu.au
}

See next page for additional authors

Follow this and additional works at: https://ro.uow.edu.au/eispapers1

Part of the Engineering Commons, and the Science and Technology Studies Commons

Research Online is the open access institutional repository for the University of Wollongong. For further information contact the UOW Library: research-pubs@uow.edu.au 


\title{
Effect of multi-walled carbon nanotubes on the cross-linking density of the poly(glycerol sebacate) elastomeric nanocomposites
}

\author{
Abstract \\ Processing conditions deeply affect the mechanical, chemical and biological properties of elastomeric \\ based nanocomposites. In this work, multi-walled carbon nanotubes (MWCNTs) were dispersed in \\ poly(glycerol sebacate) (PGS) prepolymer, followed by curing under vacuum at $120^{\circ} \mathrm{C}$. It was observed an \\ increase of the water contact angle with the amount of MWCNTs added, as well as the tensile strength \\ and Young modulus, without compromising the elastomeric behaviour of the pristine PGS matrix. The \\ cross-linking degree was determined by the Flory-Rehner swelling method and through the mechanical \\ rubber elasticity model, and an increase of more than six-fold was observed, which demonstrates the \\ chemical conjugation between the MWCNTs and the PGS polymer chains, resulting in stiff and \\ elastomeric nanocomposites. Finally, in vitro cell culture of adult mouse hypothalamus neurons A59 cells \\ showed good support for cell viability and stimulation for axons and dendrites growth. The unique \\ features of these nanocomposites make them promise for biomedical applications, as soft tissue \\ substrates with tailored mechanical properties.

\section{Disciplines} \\ Engineering | Science and Technology Studies

\section{Publication Details} \\ Yan, Y., Sencadas, V., Jin, T., Huang, X., Lie, W., Wei, D. \& Jiang, Z. (2018). Effect of multi-walled carbon \\ nanotubes on the cross-linking density of the poly(glycerol sebacate) elastomeric nanocomposites. \\ Journal of Colloid and Interface Science, 521 24-32.
}

\section{Authors}

Yi Yan, Vitor Sencadas, Tiantian Jin, Xu-Feng Huang, Wilford Lie, Dongbin Wei, and Zhengyi Jiang 


\section{Effect of multi-walled carbon nanotubes on the cross-linking density of the poly(glycerol sebacate) elastomeric nanocomposites}

Yi Yan ${ }^{1}$, Vitor Sencadas ${ }^{1,2 *}$, Tiantian $\mathrm{Jin}^{3}$, Xufeng Huang ${ }^{3}$, Wilford Lie ${ }^{4}$, Dongbin Wei ${ }^{1}$, Zhengyi Jiang ${ }^{1, *}$

*Corresponding author: Vitor Sencadas (victors@uow.edu.au) and Zhengyi Jiang (jiang@uow.edu.au)

${ }^{1}$ School of Mechanical, Materials, Mechatronic and Biomedical Engineering, University of Wollongong, Wollongong, NSW 2522, Australia

${ }^{2}$ ARC Center of Excellence for Electromaterials Science, University of Wollongong, 2522 NSW, Australia

${ }^{3}$ Illawarra Health and Medical Research Institute, University of Wollongong, Wollongong, NSW 2522, Australia

${ }^{4}$ School of Chemistry, University of Wollongong, Wollongong, NSW 2522, Australia

Abstract: Processing conditions deeply affect the mechanical, chemical and biological properties of elastomeric based nanocomposites. In this work, multi-walled carbon nanotubes (MWCNTs) were dispersed in poly(glycerol sebacate) (PGS) prepolymer, followed by curing under vacuum at $120{ }^{\circ} \mathrm{C}$. It was observed an increase of the water contact angle with the amount of MWCNTs added, as well as the tensile strength and Young modulus, without compromising the elastomeric behaviour of the pristine PGS matrix. The cross-linking degree was determined by the Flory-Rehner swelling method and through the mechanical rubber elasticity model, and an increase of more than six-fold was observed, which demonstrates the chemical conjugation between the MWCNTs and the PGS polymer chains, resulting in stiff and elastomeric nanocomposites. Finally, in vitro cell culture of adult mouse hypothalamus neurons A59 cells showed good support for cell viability and stimulations for axons and dendrites growth. The unique features of these nanocomposites make them promise for biomedical applications, as soft tissue substrates with tailored mechanical properties. 
Keywords: Carbon nanotubes, poly(glycerol sebacate), nanocomposites, biocompatibility, biomedical applications

\section{Introduction}

Carbon nanotubes (CNTs) are widely used for different applications, such as supercapacitors [1], lithium batteries [2], wearable devices [3], electrochemical biosensors [4] and biomedical engineering [5], due to its extraordinary mechanical, electrical and thermal properties.

CNTs are known to enhance the thermal and mechanical properties of polymer based composites [6]. Additionally, they are easily dispersed in liquid media by ultrasound techniques, and due to their intrinsic electrical properties, they are widely used induce electrical conductivity to insulator materials $[6,7]$.

Owing to its unique features, the blending of CNTs with polymeric matrices allows the development of nanocomposites with enhanced properties when compared to the initial raw materials. For instance, the electrical conductivity can change dramatically, within several orders of magnitude, by adding a small amount of CNTs to the insulating polymer matrix [8]. Since the work of Ajayan et al. [9] in 1994, numerous polymers were reported for the manufacture of carbon nanotube-polymer based composites, including epoxy [9], polystyrene [10], polypropylene [11], poly(methyl methacrylate) [12], polyurethane, polyimide [13], among other soft matrix materials. With the introduction of carbon nanotubes into the polymer matrix, the physical properties (e.g. mechanical, and electrical) could be enhanced, especially when chemically functionalised fillers are used [6].

Electrically conductive composites have great potential as flexible electrodes in displays, electronic paper, motion-detected devices [14] or even for biomedical engineering purposes. Collagen [15], chitosan [5], poly(propylene fumarate) [16], among other biocompatible polymeric materials blended with MWCNTs, showed positive results as suitable substrates for tissue engineering applications. The diameters of carbon nanotubes are ranging from $1 \mathrm{~nm}$ (single-walled carbon nanotubes) to $10-100 \mathrm{~nm}$ (multi-walled carbon nanotubes), which are similar to those of small nerve fibers, synaptic contacts and growth cone filopodia [17], providing the morphological clue and support for neuron adhesion and long term survival [18]. Good electrical conductivity could lead to the formation of neuronal circuits and related stimulation [19, 20], making carbon nanotubes excellent candidate for nerve tissue repair [18]. A homogeneous distribution of the MWCNTs among the polymer matrix is usually associated to mechanical [21] and electrical properties enhancement of the overall nanocomposites. Different methods have been reported in the preparation of the MWCNT- 
polymer composites, including solution mixing, in-situ polymerisation, melt blending and chemical treatment [7]. Solution mixing shows better efficiency in separating the carbon bundles, reducing cluster size, promoting a more homogeneous dispersion, which enhances the mechanical and electrical properties of the nanocomposites [14, 22].

Poly(glycerol sebacate) (PGS) is a tough biodegradable elastomer, synthesised by polycondensation of glycerol and sebacic acid [23]. The starting materials of PGS are safe for in vivo applications, as glycerol has been approved to be used in foods and sebacic acid is a natural human metabolic intermediate [24]. The mechanical properties and degradation kinetics of PGS could be tailored by controlling the curing time and temperature [25]. Moreover, PGS is a biocompatible and biodegradable elastomer used in soft tissue engineering applications, i.e. heart muscle [25], vascular tissue [26], cartilage [27], retina [28], nerve conduits [29].

Nanocomposites of multi-walled carbon nanotubes-poly(glycerol sebacate) (MWCNT-PGS) showed that the incorporation of the fillers increased the overall mechanical stiffness without compromising significantly the elasticity [30], which is an important feature for soft tissue applications [31]. In this work, MWCNT-PGS nanocomposites with different filler loadings were synthesised by solution mixing and two-step polycondensation synthesis of PGS. The chemical functional groups and molar mass of PGS prepolymer was studied by nuclear magnetic resonance (NMR). After curing, nanocomposites morphological, wettability, mechanical and chemical properties were systematically assessed. The influence of the filler in the polymer cross-linking density was studied by Flory-Rehner theory and correlated with the rubber elasticity theory. Finally, the suitability of the developed nanocomposites for biomedical applications was explored through in vitro biological assays of A59 neuron cell line.

\section{Materials and Methods}

2.1. Synthesis of the nanocomposites: The MWCNT-PGS nanocomposites were prepared following the reported methods [23, 30]. Briefly, PGS prepolymer was prepared by mixing equimolar of glycerol ( $\geq 99.5 \%$, Sigma Aldrich) and sebacic acid (> 95\% GC, Sigma Aldrich) at $120{ }^{\circ} \mathrm{C}$ under argon for $24 \mathrm{~h}$. Pristine PGS matrix was obtained by pouring PGS prepolymer into a poly(tetrafluoride) (PTFE) based mould and placed inside an oven at $120{ }^{\circ} \mathrm{C}$ under 100 mTorr vacuum (Vacuo-Temp, Selecta), during 48 h. 
Nanocomposites of PGS with MWCNTs (Play with Carbon Pty) were prepared by dispersing the filler in tetrahydrofuran (THF, $\geq 99.9 \%$, Sigma-Aldrich) with the help of an ultrasound bath (Bandelin, Sonorex Super RK106) during $6 \mathrm{~h}$, followed by addition of the desired amount of PGS prepolymer. The nanocomposite solution was them placed on top of a magnetic stirrer (Velp) until complete dissolution. The amount of solvent related to the prepolymer was $1 \mathrm{~g}$ of PGS for $5.5 \mathrm{ml}$ of solvent. The amount of MWCNTs on the elastomeric matrix ranged from 0 and $3 \mathrm{wt} \%$. After complete dissolution of the PGS prepolymer, the solution was poured into a PTFE mould, and placed in a fume cupboard overnight to allow complete solvent evaporation, followed by the curing procedure described previously for PGS matrix.

2.2. Material Characterisation: NMR (nuclear magnetic resonance) spectra, including 1D 1H, 1D 13C, 2D COSY (correlation spectroscopy), 2D TOCSY (total correlation spectroscopy), and 2D HSQC (heteronuclear single-quantum correlation spectroscopy), of the prepolymer in deuterated chloroform $(\mathrm{CDCl} 3,99.8$ atom \%, with $0.1 \%$ v/v tetramethylsilane, Sigma-Aldrich) were collected with a Varian Inova 500-MHz NMR spectrometer at $25{ }^{\circ} \mathrm{C}$. The molar weight of the prepolymer was measured by Shimadzu UFLC system equipped RID-10A and a Sedere ELSD detector, at room temperature using THF at a flow rate of $1 \mathrm{ml} / \mathrm{min}$ on Phenomenex Phenogel column (00k-0644-k0).

The nanocomposite samples were fractured after immersion in liquid nitrogen for $30 \mathrm{~min}$. Platinum coating of around $10 \mathrm{~nm}$ was deposited on the cross section of each sample by sputtering (Dynavac Sputter Coater) and scanning electron microscope (SEM) images were taken by JEOL 7500 field emission scanning electron microscope.

Contact angle measurements (sessile drop in dynamic mode) were performed at room temperature in a Data Physics OCA20 device using ultrapure water as test liquid. A sessile drop $(2 \mu \mathrm{L})$ of ultrapure water (Milli-Q, Millipore) was deposited on the surface of the samples and the contact angle was measured using a DataPhysics OCA 20 goniometer and SCA20 software. For each sample, contact angles were measured on 8 different spots and the result was expressed as their average and standard deviation. Infrared measurements (FTIR) were performed at room temperature (IRAffinity-1S, Shimadzu). Spectra were collected after 32 scans and with a resolution of $2 \mathrm{~cm}^{-1}$, from 4000 to $650 \mathrm{~cm}^{-1}$.

Mechanical measurements were performed in tensile mode on dog-bone shape samples with a gauge length of $18 \mathrm{~mm}, 2 \mathrm{~mm}$ width and $1 \mathrm{~mm}$ thick, in a universal testing machine (EX-L, Shimadzu) with a load cell of $10 \mathrm{~N}$, at a testing rate of $1 \mathrm{~mm} / \mathrm{min}$. All experiments were 
performed at room temperature $\left(\sim 22{ }^{\circ} \mathrm{C}\right)$. The mechanical parameters were obtained as the average of five individual measurements.

Nanocomposites X-ray patterns were collected in an X-ray diffraction instrument (GBC MMA X-Ray diffraction unit) with $\mathrm{CuK}_{\alpha}$ monochromatic radiation at $40 \mathrm{kV}, 30 \mathrm{~mA}$ and $\lambda=$ $1.5406 \AA$, at room temperature. The relative intensity was recorded at a scattering range of $2 \theta=10-100^{\circ}$ with a step size of $2 \theta=0.040^{\circ}$ and a step time of $10 \% \mathrm{~min}$. Raman spectra were obtained by the Horiba Jobin Yvon LabRAM HR 800 Raman spectrometer using excitation of $\mathrm{He}-\mathrm{Ne} 632.8 \mathrm{~nm}$ with a power of $13.5 \mathrm{~mW}$, and a spectral resolution of $2 \mathrm{~cm}^{-1}$.

\subsection{Elastomer swelling and crosslinking density}

The density of the pristine PGS and nanocomposite samples with different loadings of MWCNTs was measured by the pycnometer using ultrapure water. To decrease the effect of any air bubble present on density, the pycnometer was sonicated during $10 \mathrm{~min}$, and then placed under vacuum (Vacuo-Temp, Selecta). After $10 \mathrm{~min}$, the samples density was measured.

For swelling experiments, the samples were immersed in THF and weight every day (EX125D, OHAUS) until reaching a constant weight: this was normally achieved after $48 \mathrm{~h}$ immersed in the liquid. Excessive solvent on surfaces of the sample was removed gently by blotting with filter paper, followed by sample weighting and storage in a sealed glass vial, to prevent solvent evaporation. After each individual weighting, the samples were placed in a vacuum chamber (Vacuo-Temp, Selecta) during $48 \mathrm{~h}$ at $30{ }^{\circ} \mathrm{C}$, to evaporate all the solvent and reach a constant mass value. The weight of the samples before and after swelling was compared and no weight loss was detected. The swelling degree was calculated as follows:

$$
\text { Swelling degree }(\%)=\left(\frac{m_{e q}-m_{d}}{m_{d}}\right) \times 100
$$

where $m_{e q}$ is the swollen mass and $m_{d}$ is the initial dry mass. Each value was averaged from three independent measurements.

\subsection{Cell culture and viability}

Samples were cut into circular discs $(\varnothing=6.4 \mathrm{~mm})$ and immersed overnight in phosphatebuffered saline (PBS, $\mathrm{pH}=7.4$ ), with mild mechanically stirring. Prior to cell seeding, 
samples were sterilised with ultra-violet light $(\lambda=254 \mathrm{~nm})$ during $20 \mathrm{~min}$. Adult mouse hypothalamus neurons A59 cell line (mHypoA-59, Cellutions biosystems) was used to evaluate the in vitro biocompatibility of PGS nanocomposites. Samples were placed on the bottom of a 96 well plate and 1000 cells were seeded for each well.

Cells were incubated in $100 \mu \mathrm{l}$ Dulbecco's modified Eagle medium (DMEM) (D5796, Sigma-Aldrich) at $37^{\circ} \mathrm{C}$ and $5 \% \mathrm{CO}_{2}$ for 3 days. Cell culture medium was refreshed on day 2. Methotrexate (MTX) assay was applied on day 1 and day 3 to assess the cell viability. Briefly, $20 \mu \mathrm{L}$ of the MTX solution was added into each well and then the plate was incubated at $37{ }^{\circ} \mathrm{C}, 5 \% \mathrm{CO}_{2}$ in humidified environment for $2 \mathrm{~h}$, after which cell viability was measured with the help of a Spectromax plus 384. Cell viability after 1 and 3 days was obtained by comparing the optical density values of the nanocomposites groups and control PGS group. Cell growth and morphology were also evaluated by SEM. On day 1 and 3 cells were fixed with $4 \%$ paraformaldehyde in Dulbecco's PBS for $2 \mathrm{~h}$ at room temperature. Samples were then put into the liquid nitrogen during $30 \mathrm{~min}$, to freeze and a JEOL 6490LV SEM was used to observe cell morphology.

\subsection{Statistics and Data Analysis}

One-way analysis of variance (ANOVA) with Turkey's post-test was carried out to compare the means of different data sets within each experiment in IBM SPSS Statistics 22 software. A value of $p \leq 0.05$ was considered statistically significant.

\section{Results and discussion}

\subsection{PGS pre-polymer characterisation}

The analysis of the NMR spectra resulted in the complete assignments of all proton peaks (Fig. 1). Four compounds, labelled respectively by A, B, C and D in Fig. 1a, were found in the NMR sample. The methylene groups of the sebacic acid parts of compounds A and B were assigned to Peak 5, 6 and 7, which had an integration ratio of roughly 4:4:8, implying that the average of $m$ (Fig. 1a) in the sebacic acid parts of the polymers was about 4 . The glycerol part of the ${ }^{1} \mathrm{H}$ spectrum was completed assigned by using various 2D NMR spectra. These results were presented on an expansion of a TOCSY spectrum in Fig. 1b, where four TOCSY spin systems were shown by the dash boxes. The heteronuclear multiple-bond correlation (HMBC) showed significant correlations of the carbonyl carbons with the three peaks around 5 ppm, which were assigned to I2, TA2 and TB2 for the CH's at position 2 of 
the inner parts of the polymers (A and B), the terminal part of compound $\mathrm{A}$, and the terminal part of compound B, respectively. The assignments of I1.1, I1.2, I3.1, I3.2, TA1.1, TA1.2, and TA3 were based on their TOCSY correlations with I2 and TA2, respectively. The assignments of TB1, TB2, TB3.1 and TB3.2, however, were based on their unambiguous COSY correlations. On the other hand, HMBC showed no correlation of any carbonyl carbons with $\mathrm{C} 2, \mathrm{D} 2$, and $\mathrm{TB} 2$ ', and hence $\mathrm{C} 1, \mathrm{C} 2, \mathrm{C} 3.1$ and $\mathrm{C} 3.1$ were assigned to the glycerol dimer; D1.1, D1.2 and D2 were assigned to the remaining raw glycerol (which were like those observed on the $1 \mathrm{D}$ proton spectrum of raw glycerol in $\mathrm{CDCl}_{3}$ ), and TB1' TB2' and TB3', to the terminal glycerol of polymer B. Also, based on the integration ratio of TA2 and TB2, the ratio of polymers A and B in the sample was found to be 3.6:1.0. Our result is in accordance with the findings of Aydin et al., for the microwave-assisted PGS synthesis [32]. Furthermore, APT and HSQC showed no evidence of the presence of $\mathrm{CH}_{3}$ in the sample, which implied that both ends of the compound A and B were terminated by OH's. It was then concluded that the prepolymers were products of the polycondensation of glycerol and sebacic acid [33, 34]. Moreover, PGS prepolymer average molecular weight $\left(M_{n}\right)$ of 22,000 Dalton was determined with a polydispersity index (PDI) of 1.4.

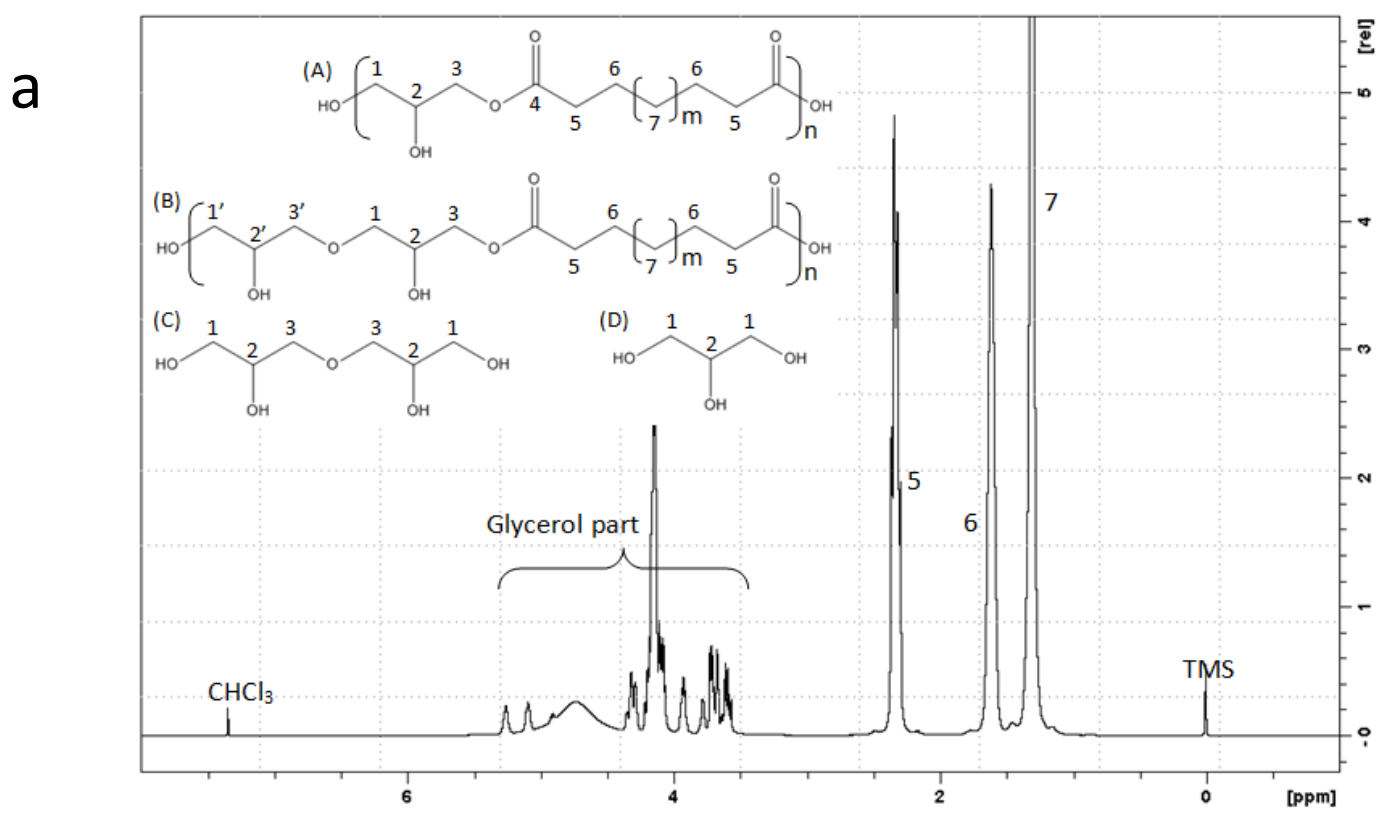




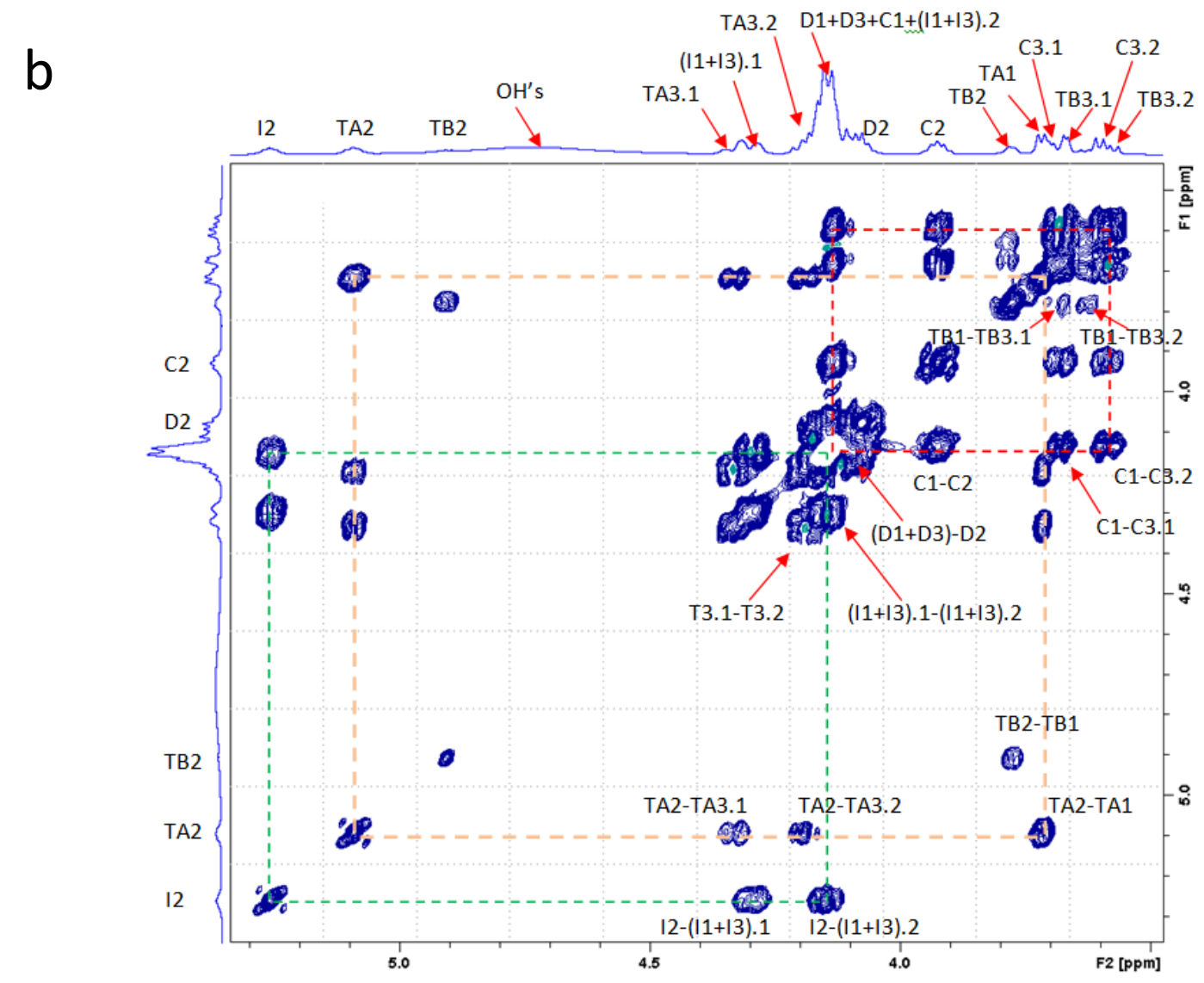

Fig. 1. ${ }^{1} \mathrm{H}$ NMR assignments of the PGS prepolymers: (a) Peak 5, 6 and 7 are the sebacic acid part of compounds (A) and (B). The glycerol part, including compounds (C) and (D), is assigned separately using a few 2D NMR spectra, as shown in (b), where peaks labelled with an 'I' (such as I1, I2 and I3) are for the internal glycerol part of the polymers, those with a ' $\mathrm{T}$ ' are the terminal part of the polymers, and those with A, B, C, D refer to the four compounds in (a).

\subsection{Nanocomposite morphology and surface properties}

The polymeric matrix presented a smooth cross-section (Fig. 2), without the presence of any air bubbles that could be entrapped in the elastomeric bulk. Samples with different amount of MWCNTs showed that the filler is homogeneously distributed among the polymeric matrix with only small filler clusters detected, even for the higher concentrations of MWCNTs (Fig. 2). 

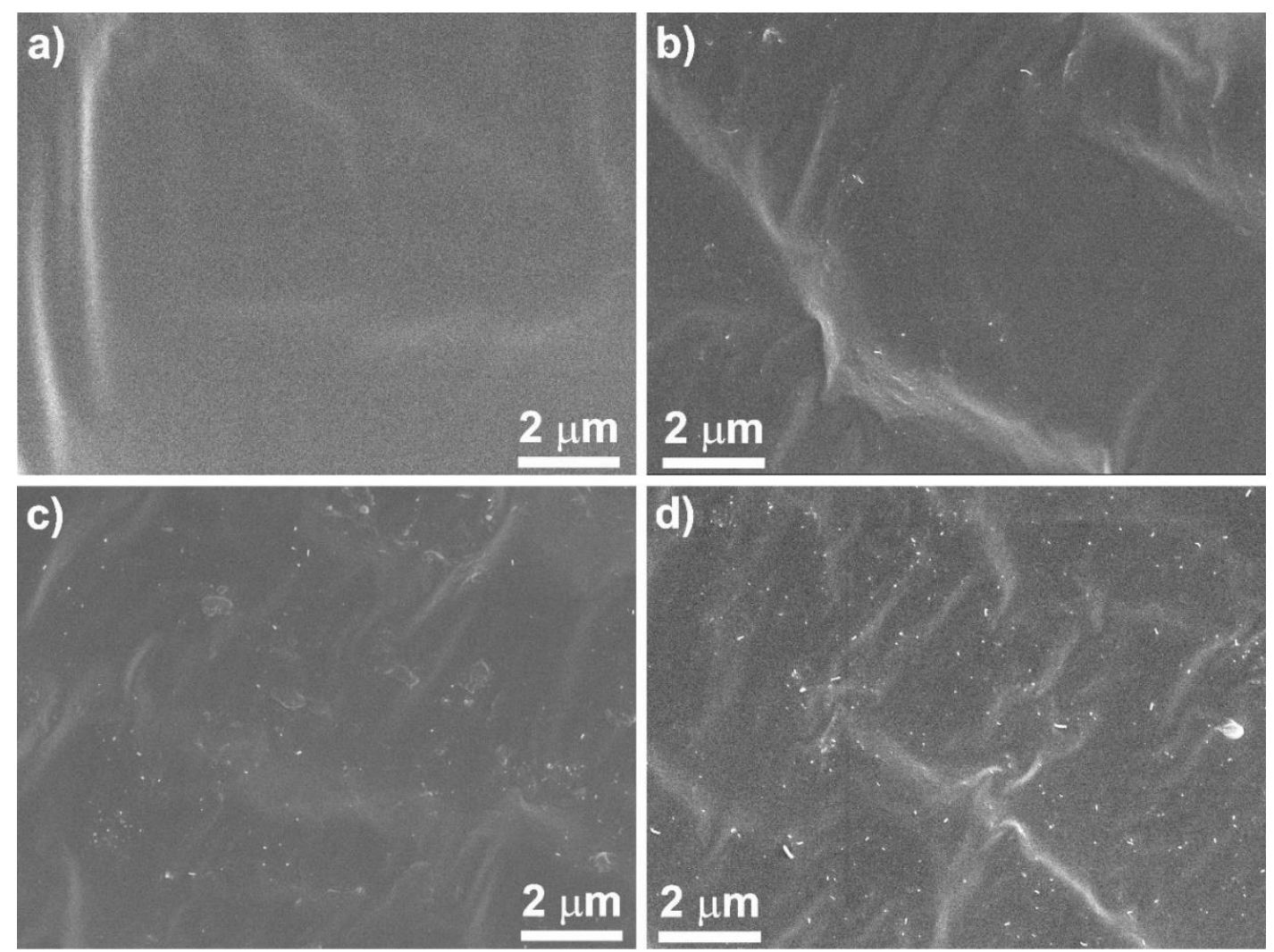

Fig. 2. Morphology of the PGS nanocomposites cross-section: (a) Pristine PGS, (b) 1 wt\% MWCNT-PGS, (c) $2 \mathrm{wt} \%$ MWCNT-PGS, and (d) 3 wt $\%$ MWCNT-PGS.

The surface properties of PGS elastomeric nanocomposites were studied through the measurement of the water contact angle (WCA). The average WCA of the PGS pristine films showed a value of $42 \pm 3^{\circ}$, which reveals a hydrophilic character for the PGS [34-36]. When MWCNTs were added, an increase of the WCA was observed (Fig. 3a), reaching a WCA of $82 \pm 3^{\circ}$ for the sample with $3 \mathrm{wt} \%$ of filler, which probably is due to changes in sample surface roughness, but also to the esterification (figure $3 b$ ) of the carboxyl groups of MWCNTs with the hydroxyl groups of PGS [30], leading to a reduced water uptake ability of the nanocomposites (Fig. 3a).

It is known that hydrophobic surfaces will reduce protein absorption and cell adhesion, however, it is also necessary to consider specific structural features, e.g. surface roughness, mechanical properties or hydrogen bonding [37]. Nevertheless, some hydrophilic surfaces with a low WCA, such as synthetic hydrogels from poly(hydroxyethyl methacrylate) [38] or natural hydrogels of alginate [39], still resist at some extent, to protein and cell attachment, especially for short-term incubation periods [37]. The WCA is then only an indicator of cell adhesion and attachment at specific surfaces. In this work, despite the increase of the WCA 
with the presence of the MWCNTs, all samples still present hydrophilic behaviour, which should favour cell adhesion and proliferation.
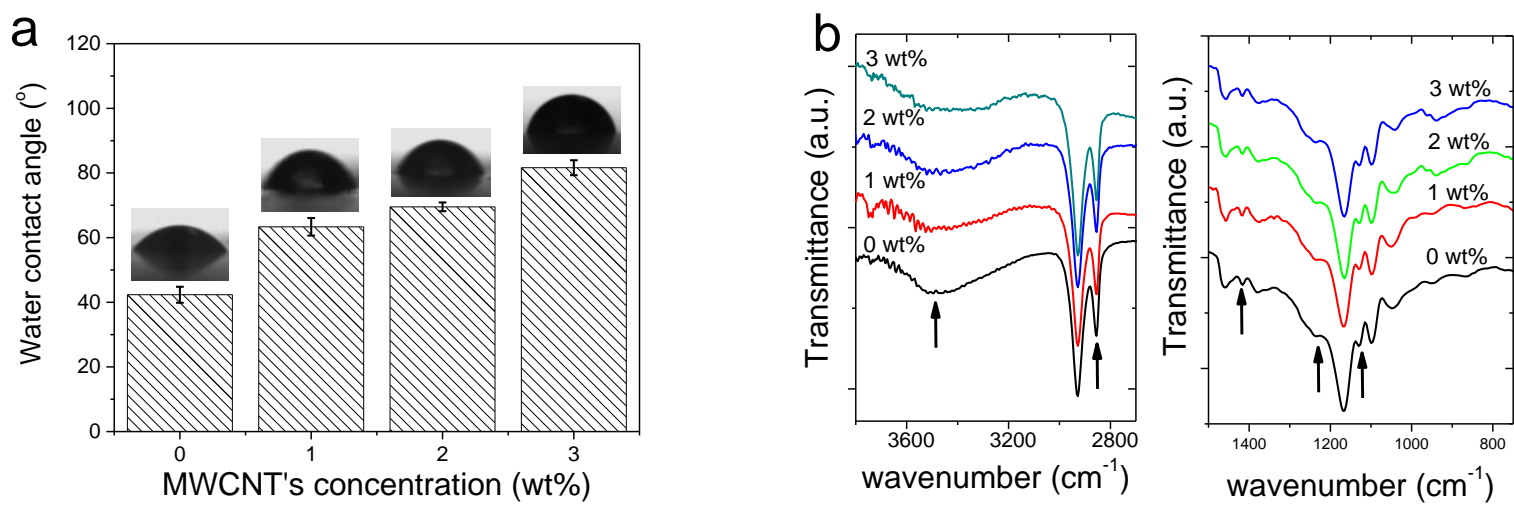

Fig. 3. (a) Evolution of the water contact angle for the MWCNT-PGS nanocomposites, and (b) FTIR spectra of the MWCNT-PGS nanocomposites.

\subsection{Chemical, Physical and Thermal properties of the MWCNT-PGS nanocomposites}

Nanocomposite structures were analysed using XRD, and the obtained spectra is presented in Fig. 4a. All samples presented similar spectra with a broad diffraction peak around $20^{\circ}$, characteristic of amorphous materials [31].

Raman spectra for PGS and MWCNT-PGS composites with different MWCNTs content were obtained to determine the interactions between the filler and the elastomeric matrix (Fig. 4b). Nanocomposite samples display two characteristic absorption bands at $1360 \mathrm{~cm}^{-1}$ assigned to the distorted or D-band, and corresponds to the impurities of defects of the MWCNTs, while the absorption band at $1580 \mathrm{~cm}^{-1}$, corresponding to the graphite of G-band, and derives from the graphite-like in-plane mode [40]. The disorder of carbon nanofillers is usually made by analysing the $I_{D} / I_{G}$ intensity ratio between the disorder D-band and the Raman allowed G-band (Fig. 4c). The incorporation of MWCNTs into the elastomeric matrix leads to a decrease of the $I_{D} / I_{G}$ intensity ratio (Fig. 4c), suggesting that the structure of the filler was distorted due to the chemical interaction of the MWCNTs with the PGS chains during the curing procedure, probably enhancing crosslinking density [30, 41].

Thermalgravimetric analysis (TGA) of the PGS nanocomposites showed that the thermal degradation profile of PGS does not suffer significant changes with the incorporation of the MWCNTs (Fig 4d). Additionally, the onset degradation temperature, calculated by extending the pre-degradation portion of the TGA curve to the point of interception with a line drawn as 
a tangent to the steepest portion of the mass curve occurring during degradation [42], was found to be similar between the pristine PGS and the MWCNT-PGS nanocomposite samples. Furthermore, it was observed that the residual mass, calculated at $600{ }^{\circ} \mathrm{C}$, increases with the amount of filler placed initially into the PGS pre-polymer (inset of Fig. 4d).
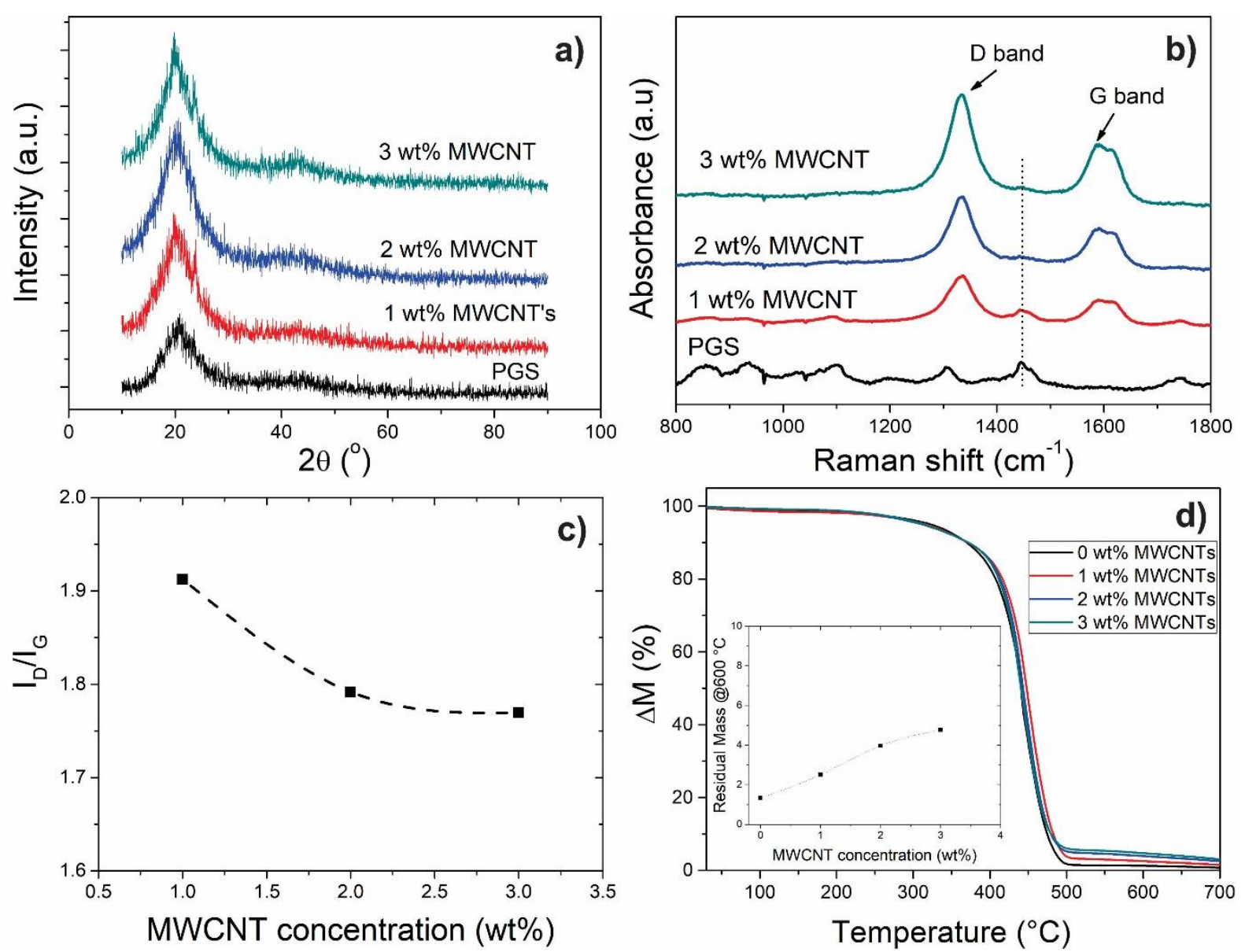

Fig. 4. (a) X-ray diffraction, (b) Raman spectra for PGS, 1 wt $\%$ MWCNT-PGS, 2 wt\% MWCNT-PGS and 3 wt\% MWCNT-PGS, (c) D-band and G-band intensity ratio for Raman spectra, and (d) Thermal degradation profile for the MWCNT-PGS samples, recorded at $20{ }^{\circ} \mathrm{C} / \mathrm{min}$ (inset shows the evolution of the sample residue at $600{ }^{\circ} \mathrm{C}$ ).

\subsection{Evaluation of nanocomposites cross-linking density}

Representative quasi-static stress-strain measurements for the MWCNT-PGS nanocomposites are illustrated in Fig. 5a. It was observed that the incorporation of the filler increases the ultimate stress strength (UTS) and leads to a decrease of the strain at break ( $\left.\varepsilon_{\text {break }}\right)$ (Fig. $\left.5 b\right)$. Further, the Young's modulus $(E)$ of the PGS elastomer was $129 \pm 46 \mathrm{kPa}$, and the addition of MWCNTs resulted in a more than six-fold increase in the elastic modulus up to $858 \pm 95$ $\mathrm{kPa}$, compared to the pristine polymer matrix (Table 1). The enhancement of mechanical 
properties is probably due to an increase of cross-linking density due to the presence of the MWCNTs, that restricts polymer chain movement during the uniaxial mechanical loading (Fig 5). Gaharwar et al. [30] suggested that the increase of the nanocomposite mechanical performance was due to the covalent bond formed between the filler and the backbone of the polymer chains.
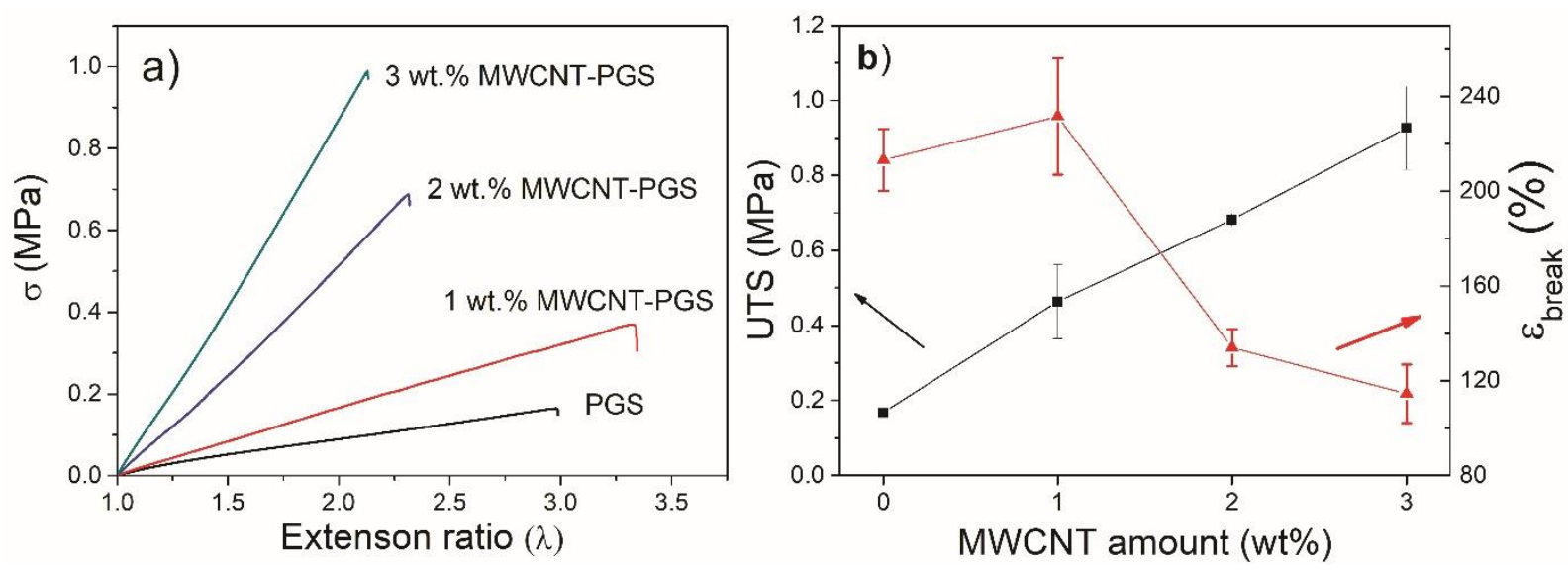

Fig. 5. (a) Tensile strain behaviour and (b) ultimate tensile strength (UTS) and strain at break $\left(\varepsilon_{\text {break }}\right)$ for PGS nanocomposites with different amounts of MWCNTs.

Swelling degree is another method to qualitatively and quantitatively evaluate the crosslinking density of the elastomeric matrix [30, 34]. Fig. 6 shows the samples general appearance before and after swelling in tetrahydrofuran (THF), where is possible to visualise the macroscopic evolution in sample dimensions, for the PGS and MWCNT-PGS nanocomposite samples loaded with 1 wt.\% MWCNTs.

Nanocomposite samples almost double their original size, after THF uptake (Fig. 6). The swelling degree, calculated through equation 1, shows a decrease in the solvent uptake with the amount of filler added to the polymer network (Table 1), which suggests that the filler participates in the polymer network formation, providing new cross-linking sites for the PGS chains to attach, which is in accordance with the findings reported for the mechanical properties (Fig. 5) and Raman spectroscopy (Fig. 4).

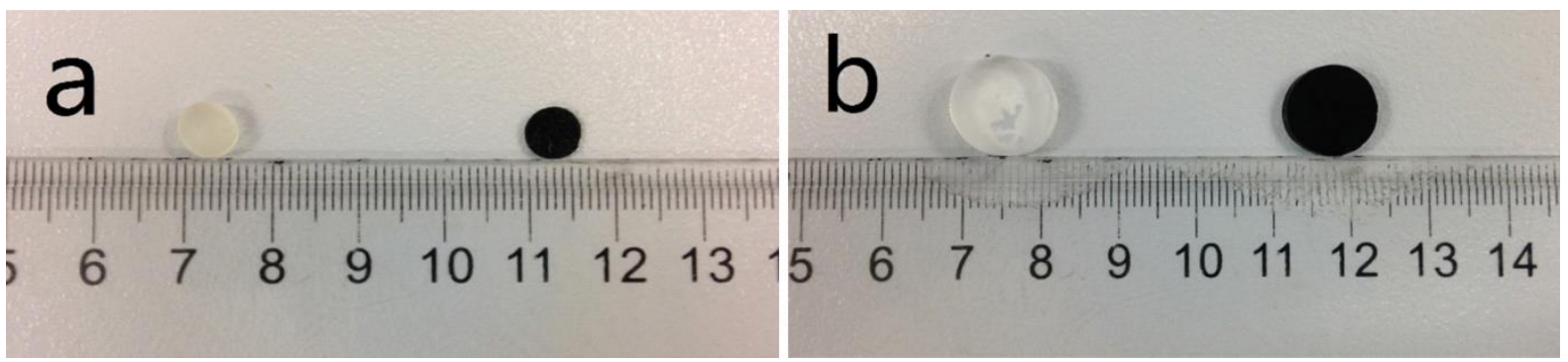


Fig. 6. Pristine and MWCNT-PGS (1 wt $\%$ filler): a) before swelling experiments and b) after immersing in THF during $48 \mathrm{~h}$.

Furthermore, the swelling behaviour of a solvent into an elastomeric network is an effective way to measure the cross-linking density promoted by the curing procedure. According to Flory [43], the density of a network chain between cross-links is given by:

$$
\mathrm{n}=\frac{1}{2} \frac{\left[\ln \left(1-v_{2}\right)+v_{2}+\chi v_{2}{ }^{2}\right]}{v_{1}\left(v_{2} / 2-v_{2}{ }^{1 / 3}\right)}
$$

where $\mathrm{n}$ is the cross-linking density, $v_{2}$ is the volume fraction of polymer in the equilibrium swollen network, $v_{1}$ is the molar volume fraction of the solvent, and $\chi$ is Flory-Huggins polymer-solvent interaction parameter [43]. For a tetra-functional affine network, the crosslinking density of the swollen material can be calculated through the Flory - Rehner theory:

$$
v_{2}=\left[1+\left(\frac{m_{e q}-m_{d}}{m_{d}}\right)\left(\frac{\rho_{2}}{\rho_{1}}\right)\right]^{-1}
$$

where, $\rho_{1}$ and $\rho_{2}$ are the solvent and polymer density, respectively. Furthermore, $v_{2}$ could be obtained through the swelling degree measurements performed for the different samples (Table 1).

For elastomeric materials, the cross-linking density is also related with $E$ through [44]:

$$
E=3 R T n
$$

where, $R$ is the universal gas constant and $T$ is the absolute temperature.

Combining the Eq. 2 and 4, the Flory-Huggins parameter $(\chi)$ can be determined by:

$$
\frac{E}{3 R T}=\frac{1}{2} \frac{\left[\ln \left(1-v_{2}\right)+v_{2}+\chi v_{2}{ }^{2}\right]}{v_{1}\left(v_{2} / 2-v_{2}{ }^{1 / 3}\right)}
$$

According to the model developed above, the cross-linking density can be obtained through Young modulus and swelling degree of the elastomeric nanocomposites (Table 1).

The cross-linking density calculated by the rubber elasticity model (Eq. 4), showed that the number of cross-links present in the PGS elastomer ranged from $17.4 \pm 6.2 \mathrm{~mol} / \mathrm{m}^{3}$ for the 
neat polymer matrix, increasing up to $115.5 \pm 12.8 \mathrm{~mol} / \mathrm{m}^{3}$ for the nanocomposite sample with 3 wt $\%$ MWCNTs.

The Flory-Huggins polymer-solvent interaction parameter is a constant for a specific pair of solvent and polymer, and does not change with the cross-linking density of the polymer chain [45]. In our work, the value of $\chi$ was determined through Eq. 5, and a value of 0.43 was found, which is in accordance to the values previously reported for the PGS-THF system [31]. When MWCNTs are added to the PGS matrix, a deviation between the cross-linking density calculated through Eq. 2 and the mechanical theory (Eq. 4) was observed with the increasing amount of the filler added to the elastomeric matrix (Table 1). Taking into consideration that the $\chi$ does not change for the same polymer-solvent system [45], and the only parameter changed was the filler amount added before the curing of the elastomeric matrix, one may conclude that the MWCNTs are covalently bonded to the PGS polymer chains, increasing the cross-linking density of the elastomeric matrix, which correlates with the Raman spectroscopy results (Fig. $4 \mathrm{~b}$ and c).

Flory-Rehner theory is based on the swelling behaviour of the elastomeric matrix and does not require the alignment of the polymer chains, thus it only considers the chemical crosslinks. Real polymer networks contain both the chemical and physical cross-links, being the later related with the various kinds of entanglements present in the elastomeric matrix and could explain the difference obtained for the cross-linking density calculated through the two methods, i.e. Flory-Rehner and mechanical rubber elasticity theory (Table 1). Although, the exact nature of this effect is not fully understood, and more work is needed to understand this effect.

Mechanical properties of elastomers are deeply influenced by the cross-linking density. This study showed that it is possible to engineer the PGS mechanical behaviour by incorporation of an active filler, capable to create new sites to attach the polymeric chains, and increase the cross-linking density of the soft elastomer. A pathway to understand the effect of the filler in the overall mechanical properties was developed, and it provides new insights to understand the role of the MWCNTs during the curing procedure of the PGS elastomer. However, a comprehensive understanding of the individual contribution of the physical and chemical cross-links to the overall mechanical response of the polymer matrix is still a challenge. 
Table 1. MWCNT-PGS physical parameters.

\begin{tabular}{|c|c|c|c|c|}
\hline $\begin{array}{c}\text { MWCNT } \\
\text { loading } \\
(w t \%)\end{array}$ & $\begin{array}{c}\text { Swelling } \\
\text { degree } \\
(\%)\end{array}$ & $\begin{array}{c}n \\
\text { (swelling method, } \chi \\
=0.43, \mathrm{~mol} / \mathrm{m}^{3} \text { ) }\end{array}$ & $\begin{array}{c}E \\
(\mathbf{k P a})\end{array}$ & $\begin{array}{c}n \\
(\text { mechanical } \\
\left.\text { method, } \mathbf{m o l} / \mathbf{m}^{3}\right)\end{array}$ \\
\hline 0 & $760 \pm 10$ & $16.8 \pm 0.8$ & $129 \pm 46$ & $17.4 \pm 6.2$ \\
\hline 1 & $675 \pm 13$ & $19.1 \pm 0.2$ & $220 \pm 15$ & $29.6 \pm 2.0$ \\
\hline 2 & $526 \pm 18$ & $28.4 \pm 1.2$ & $492 \pm 85$ & $66.1 \pm 11.6$ \\
\hline 3 & $435 \pm 10$ & $38.2 \pm 1.3$ & $858 \pm 95$ & $115.5 \pm 12.8$ \\
\hline \multicolumn{5}{|c|}{$n$-Cross-linking density } \\
\hline \multicolumn{5}{|c|}{$E$ - Mechanical Young Modulus } \\
\hline \multicolumn{5}{|c|}{$\chi-$ Flory-Huggins polymer-solvent interaction parameter } \\
\hline
\end{tabular}

\subsection{Cell culture and viability}

Cell viability on PGS and nanocomposite groups at day 1 and day 3 was measured using MTX assay. As illustrated in Fig. 7, on day 1 there was no difference between PGS and nanocomposite samples, indicating that the tested nanocomposites do not affect the initial cell adhesion. After three days, the cell viability was significantly increased in all groups, being the nanocomposite group significantly higher when compared to pristine PGS. However, the differences among nanocomposite samples for different MWCNTs loadings were negligible. SEM analysis strongly corroborates these results. Since phenotypic features of cells were similar among the several samples, only $2 \mathrm{wt} . \%$ MWCNT-PGS nanocomposite was selected to show the morphology of neurons in Fig. 7. After 3 days, samples presented high cell density on their surfaces where axons and dendrites of about $50 \mu \mathrm{m}$ length could be observed, indicating the outgrowth support. Overall, these results suggest that the elastomeric nanocomposites presented better cytocompatibility than PGS alone. 

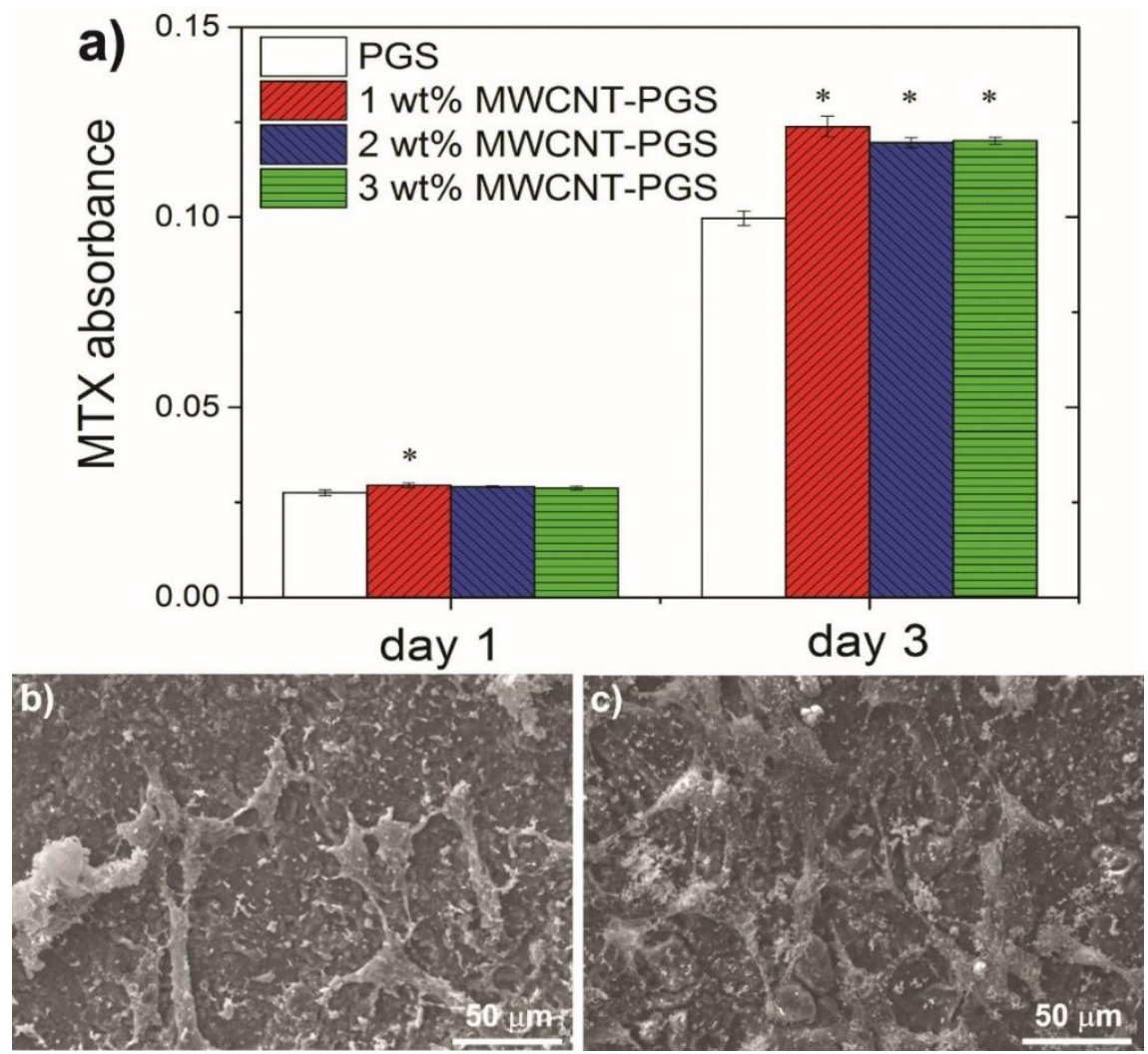

Fig. 7. (a) Cell viability of adult mouse hypothalamus neurons A59 cells cultured over PGS and MWCNT-PGS nanocomposite membranes for 1 and 3 days, estimated by MTX assay. Values are presented as the mean \pm SD of three independent experiments; statistical differences were reported as $* \mathrm{p}<0.05$. Microscopic of photographs of cells cultured during 3 days on: (b) PGS and c) MWCNT-PGS nanocomposite membranes, showing cells in advanced stage of adhesion and spreading.

\section{Conclusions}

In this study, elastomeric MWCNT-PGS nanocomposites were successfully processed, with the MWCNTs homogenously dispersed in the polymer matrix. It was observed that the incorporation of the filler leads to an increase of the material hydrophobicity. The mechanical properties of the nanocomposites showed that there is an increase of the material Young modulus when the filler is added to the PGS, due to the covalent bonds formed at the interface between the polymer chains and the MWCNTs, which ultimately led to an increase of the overall cross-linking density. The PGS-THF swelling behaviour showed that the neat PGS elastomer has a Flory-Huggins polymer-solvent interaction parameter of 0.43 , but when the MWCNTs are added to the PGS, the Flory-Rehner theory cannot accurately predict the cross-linking density of the nanocomposites, due to the amount of new cross-link spots (chemical and physical cross-links) that are created by the filler. The overall mechanical 
properties have a contribution from the chemical cross-links between the polymer chains and the filler, but physical chain entanglements promoted by the nanofiller must be considered to the mechanical performance of elastomeric nanocomposites. The biological characterisation demonstrates the in vitro biocompatibility to support neurons from hypothalamus and the outgrowth of axons and dendrites.

This work demonstrates a route to tailor and understand the effect of nanofillers in the overall mechanical properties of elastomeric matrix. It opens the opportunity to engineer new biomedical materials with tailored electrical and mechanical properties, that can withstand the surgical procedure, and to better match the properties of the native tissue.

\section{Acknowledgements}

The authors acknowledge financial support from China Scholarship Council (CSC), UPA from University of Wollongong and Australian Research Council (ARC) for Future Fellowship (Z.Y. Jiang). The authors acknowledge use of facilities within the UOW Electron Microscopy Centre and the Australian National Nanofabrication Facility - Materials node for equipment use. The authors acknowledge Mr. Jiangshan Zhang, Mr. Hui Wu and Dr. Tony Romeo for SEM imaging, Ms. Hongqin Wang, Dr. Xiaoqi Chen, Dr. Qiang Fu and Ms. Zehra Boz for help of cell culture assays.

\section{References}

[1] J. Huang, B.G. Sumpter, V. Meunier, G. Yushin, C. Portet, Y. Gogotsi, Curvature effects in carbon nanomaterials: Exohedral versus endohedral supercapacitors, Journal of Materials Research, 25 (2010) 1525-1531.

[2] B.J. Landi, M.J. Ganter, C.D. Cress, R.A. DiLeo, R.P. Raffaelle, Carbon nanotubes for lithium ion batteries, Energy \& Environmental Science, 2 (2009) 638-654.

[3] T. Yamada, Y. Hayamizu, Y. Yamamoto, Y. Yomogida, A. Izadi-Najafabadi, D.N. Futaba, K. Hata, A stretchable carbon nanotube strain sensor for human-motion detection, Nat Nano, 6 (2011) 296-301.

[4] J. Wang, Carbon-Nanotube Based Electrochemical Biosensors: A Review, Electroanalysis, 17 (2005) 7-14. 
[5] A. Abarrategi, M.C. Gutiérrez, C. Moreno-Vicente, M.J. Hortigüela, V. Ramos, J.L. López-Lacomba, M.L. Ferrer, F. del Monte, Multiwall carbon nanotube scaffolds for tissue engineering purposes, Biomaterials, 29 (2008) 94-102.

[6] J.N. Coleman, U. Khan, W.J. Blau, Y.K. Gun'ko, Small but strong: A review of the mechanical properties of carbon nanotube-polymer composites, Carbon, 44 (2006) 16241652.

[7] Z. Spitalsky, D. Tasis, K. Papagelis, C. Galiotis, Carbon nanotube-polymer composites: Chemistry, processing, mechanical and electrical properties, Progress in Polymer Science, 35 (2010) 357-401.

[8] W. Bauhofer, J.Z. Kovacs, A review and analysis of electrical percolation in carbon nanotube polymer composites, Composites Science and Technology, 69 (2009) 1486-1498. [9] P.M. Ajayan, O. Stephan, C. Colliex, D. Trauth, Aligned Carbon Nanotube Arrays Formed by Cutting a Polymer Resin—Nanotube Composite, Science, 265 (1994) 1212-1214. [10] R. Blake, J.N. Coleman, M.T. Byrne, J.E. McCarthy, T.S. Perova, W.J. Blau, A. Fonseca, J.B. Nagy, Y.K. Gun'ko, Reinforcement of poly(vinyl chloride) and polystyrene using chlorinated polypropylene grafted carbon nanotubes, Journal of Materials Chemistry, 16 (2006) 4206-4213.

[11] J. Shen, M.F. Champagne, Z. Yang, Q. Yu, R. Gendron, S. Guo, The development of a conductive carbon nanotube (CNT) network in CNT/polypropylene composite films during biaxial stretching, Composites Part A: Applied Science and Manufacturing, 43 (2012) 14481453.

[12] F. Du, R.C. Scogna, W. Zhou, S. Brand, J.E. Fischer, K.I. Winey, Nanotube Networks in Polymer Nanocomposites: Rheology and Electrical Conductivity, Macromolecules, 37 (2004) 9048-9055.

[13] Z. Ounaies, C. Park, K.E. Wise, E.J. Siochi, J.S. Harrison, Electrical properties of single wall carbon nanotube reinforced polyimide composites, Composites Science and Technology, 63 (2003) 1637-1646.

[14] M.T. Byrne, Y.K. Gun'ko, Recent Advances in Research on Carbon Nanotube-Polymer Composites, Advanced Materials, 22 (2010) 1672-1688.

[15] Y. Cho, R.B. Borgens, The effect of an electrically conductive carbon nanotube/collagen composite on neurite outgrowth of PC12 cells, Journal of Biomedical Materials Research Part A, 95A (2010) 510-517.

[16] B. Sitharaman, X. Shi, X.F. Walboomers, H. Liao, V. Cuijpers, L.J. Wilson, A.G. Mikos, J.A. Jansen, In vivo biocompatibility of ultra-short single-walled carbon 
nanotube/biodegradable polymer nanocomposites for bone tissue engineering, Bone, 43 (2008) 362-370.

[17] A. Fabbro, M. Prato, L. Ballerini, Carbon nanotubes in neuroregeneration and repair, Advanced Drug Delivery Reviews, 65 (2013) 2034-2044.

[18] M.P. Mattson, R.C. Haddon, A.M. Rao, Molecular functionalization of carbon nanotubes and use as substrates for neuronal growth, Journal of Molecular Neuroscience, 14 (2000) 175-182.

[19] V. Lovat, D. Pantarotto, L. Lagostena, B. Cacciari, M. Grandolfo, M. Righi, G. Spalluto, M. Prato, L. Ballerini, Carbon Nanotube Substrates Boost Neuronal Electrical Signaling, Nano Letters, 5 (2005) 1107-1110.

[20] A. Mazzatenta, M. Giugliano, S. Campidelli, L. Gambazzi, L. Businaro, H. Markram, M. Prato, L. Ballerini, Interfacing Neurons with Carbon Nanotubes: Electrical Signal Transfer and Synaptic Stimulation in Cultured Brain Circuits, The Journal of Neuroscience, 27 (2007) 6931-6936.

[21] M. Tarfaoui, K. Lafdi, A. El Moumen, Mechanical properties of carbon nanotubes based polymer composites, Composites Part B: Engineering, 103 (2016) 113-121.

[22] O.V. Kharissova, B.I. Kharisov, E.G. de Casas Ortiz, Dispersion of carbon nanotubes in water and non-aqueous solvents, RSC Advances, 3 (2013) 24812-24852.

[23] Y. Wang, G.A. Ameer, B.J. Sheppard, R. Langer, A tough biodegradable elastomer, Nature biotechnology, 20 (2002) 602-606.

[24] R. Rai, M. Tallawi, A. Grigore, A.R. Boccaccini, Synthesis, properties and biomedical applications of poly(glycerol sebacate) (PGS): A review, Progress in Polymer Science, 37 (2012) 1051-1078.

[25] Q.Z. Chen, H. Ishii, G.A. Thouas, A.R. Lyon, J.S. Wright, J.J. Blaker, W. Chrzanowski, A.R. Boccaccini, N.N. Ali, J.C. Knowles, S.E. Harding, An elastomeric patch derived from poly(glycerol sebacate) for delivery of embryonic stem cells to the heart, Biomaterials, 31 (2010) 3885-3893.

[26] P.M. Crapo, J. Gao, Y. Wang, Seamless tubular poly(glycerol sebacate) scaffolds: Highyield fabrication and potential applications, Journal of Biomedical Materials Research Part A, 86A (2008) 354-363.

[27] J.M. Kemppainen, S.J. Hollister, Tailoring the mechanical properties of 3D-designed poly(glycerol sebacate) scaffolds for cartilage applications, Journal of Biomedical Materials Research Part A, 94A (2010) 9-18. 
[28] C.D. Pritchard, K.M. Arnér, R.S. Langer, F.K. Ghosh, Retinal transplantation using surface modified poly(glycerol-co-sebacic acid) membranes, Biomaterials, 31 (2010) 79787984.

[29] C.A. Sundback, J.Y. Shyu, Y. Wang, W.C. Faquin, R.S. Langer, J.P. Vacanti, T.A. Hadlock, Biocompatibility analysis of poly(glycerol sebacate) as a nerve guide material, Biomaterials, 26 (2005) 5454-5464.

[30] A.K. Gaharwar, A. Patel, A. Dolatshahi-Pirouz, H. Zhang, K. Rangarajan, G. Iviglia, S.R. Shin, M.A. Hussain, A. Khademhosseini, Elastomeric nanocomposite scaffolds made from poly(glycerol sebacate) chemically crosslinked with carbon nanotubes, Biomaterials Science, (2015).

[31] Q.Z. Chen, A. Bismarck, U. Hansen, S. Junaid, M.Q. Tran, S.E. Harding, N.N. Ali, A.R. Boccaccini, Characterisation of a soft elastomer poly(glycerol sebacate) designed to match the mechanical properties of myocardial tissue, Biomaterials, 29 (2008) 47-57.

[32] H.M. Aydin, K. Salimi, Z.M.O. Rzayev, E. Piskin, Microwave-assisted rapid synthesis of poly(glycerol-sebacate) elastomers, Biomaterials Science, 1 (2013) 503-509.

[33] C.L.E. Nijst, J.P. Bruggeman, J.M. Karp, L. Ferreira, A. Zumbuehl, C.J. Bettinger, R. Langer, Synthesis and Characterization of Photocurable Elastomers from Poly(glycerol-cosebacate), Biomacromolecules, 8 (2007) 3067-3073.

[34] A. Patel, A.K. Gaharwar, G. Iviglia, H. Zhang, S. Mukundan, S.M. Mihaila, D. Demarchi, A. Khademhosseini, Highly elastomeric poly(glycerol sebacate)-co-poly(ethylene glycol) amphiphilic block copolymers, Biomaterials, 34 (2013) 3970-3983.

[35] Y. Yan, V. Sencadas, T. Jin, X. Huang, J. Chen, D. Wei, Z. Jiang, Tailoring the wettability and mechanical properties of electrospun poly(l-lactic acid)-poly(glycerol sebacate) core-shell membranes for biomedical applications, Journal of Colloid and Interface Science, 508 (2017) 87-94.

[36] Y. Yan, V. Sencadas, J. Zhang, D. Wei, Z. Jiang, Superomniphilic Poly(glycerol sebacate)-Poly(l-lactic acid) Electrospun Membranes for Oil Spill Remediation, Advanced Materials Interfaces, 4 (2017) 1700484-n/a.

[37] M.R. Alexander, P. Williams, Water contact angle is not a good predictor of biological responses to materials, Biointerphases, 12 (2017) 02C201.

[38] T.V. Chirila, I.J. Constable, G.J. Crawford, S. Vijayasekaran, D.E. Thompson, Y.-C. Chen, W.A. Fletcher, B.J. Griffin, Poly(2-hydroxyethyl methacrylate) sponges as implant materials: in vivo and in vitro evaluation of cellular invasion, Biomaterials, 14 (1993) 26-38. 
[39] I. Banerjee, R.C. Pangule, R.S. Kane, Antifouling Coatings: Recent Developments in the Design of Surfaces That Prevent Fouling by Proteins, Bacteria, and Marine Organisms, Advanced Materials, 23 (2011) 690-718.

[40] H. Aguilar-Bolados, M. Yazdani-Pedram, A. Contreras-Cid, M.A. López-Manchado, A. May-Pat, F. Avilés, Influence of the morphology of carbon nanostructures on the piezoresistivity of hybrid natural rubber nanocomposites, Composites Part B: Engineering, 109 (2017) 147-154.

[41] M.S. Dresselhaus, A. Jorio, M. Hofmann, G. Dresselhaus, R. Saito, Perspectives on Carbon Nanotubes and Graphene Raman Spectroscopy, Nano Letters, 10 (2010) 751-758. [42] D. Santos, C.O. Correia, D.M. Silva, P.S. Gomes, M.H. Fernandes, J.D. Santos, V. Sencadas, Incorporation of glass-reinforced hydroxyapatite microparticles into poly(lactic acid) electrospun fibre mats for biomedical applications, Materials Science and Engineering: C, 75 (2017) 1184-1190.

[43] P.J. Flory, Principles of polymer chemistry, Ithaca : Cornell Univ. Pr.1953.

[44] L.H. Sperling, Introduction to physical polymer science, Wiley, New York, 1992. [45] S.P. Rout, G.B. Butler, Crosslinking of diene polymers via 'ENE' reaction with bistriazolinediones, Polymer Bulletin, 2 (1980) 513-520. 